\title{
Boundary element modeling and analysis of adhesive bonded structural joints
}

\author{
G. Cavallini*, G. Davi ${ }^{* *}$, A. Milazzo** \\ *Dipartimento di Ingegneria Aerospaziale "L. Lazzarino”, Università di Pisa, \\ via Caruso 50146 Pisa - Italy \\ ** Dipartimento di Ingegneria Aeronautica e dei Trasporti, Università di Palermo, Viale \\ delle Scienze 90128 Palermo - Italy
}

\begin{abstract}
In this paper, a boundary element technique for modeling and analysis of adhesive bonded structural joints is presented. The formulation is developed in the framework of the anisotropic elasticity and attention is focused on the application to composite structural joints built with the splicing concept technique. To model and analyze composite bonded joints a multidomain implementation of the boundary element method has been used. It has been proven well suited and very effective for the characterization of the mechanical behavior of spliced joints, allowing the analysis of the high gradient stresses and strains near the splice lines as well as the prediction of the overall distribution of the interlaminar tractions. Numerical results show good agreement with analytical solution and finite element analyses.
\end{abstract}

KEY WORDS: Bonded joints, composite laminates; boundary element method.

\section{INTRODUCTION}

Due to the increased transport business, during the last decades, composite materials have been subject of growing interest in lightweight structural applications, especially in aeronautical and aerospace industries. Their inherent features, such as high specific stiffness and strength, as well as the capability of path load management, lead to remarkable structural weight savings enabling economical and environmental benefits $[1,2]$. One of the reasons for this increasing interest is given by the appealing opportunity of reducing the overall structural complexity through the manufacturing of large size panels without riveted joints. This is attained by means of emerging technologies and materials which allow low parts count with the resulting decreased number of stress concentration points, flaws, defects and thus, in general, of crack propagation sources. The development of such technologies would result in easy maintenance and in the overall reduction of manufacturing and inspection times and costs. In this framework, Fiber Metal Laminates (FMLs) have recently been subject of intensive research $[3,4]$. These hybrid materials, consisting of thin metallic sheets bonded into one laminate by intermediate fiber/resin layers, are developed with different classes like Arall ${ }^{\circledR}$ and Glare ${ }^{\circledR}[5,6]$. Much of the attention is actually focused on Glare ${ }^{\circledR}$, which consists of thin aluminum alloy sheets laminated with glass fiber reinforced prepreg layers. Different stacking sequences, aluminum properties and layer 
thicknesses lead to different FMLs grades, suitable to several applications. Moreover, the combination of the two different materials improves some of the basic properties of aluminum alloys [3, 7-10]. Due to its features Glare ${ }^{\circledR}$ is currently used for cargo floor applications of several Boeing 777 airplanes, for the lower wing panels of the Fokker 27 and it was chosen to built up the skin panels of the upper side of the fuselage of the high capacity plane A380 [11-13]. Interesting studies focused on the possibility of constructing medium and large size civil aircraft with all-composite pressurized fuselages [14]. Extensive use of Glare ${ }^{\circledR}$ in the structures of these aircrafts is estimated to lead to a $20 \%$ structural weight reduction. However, the full exploitation of the structural advantages allowed by the use of these advanced materials largely rely on the possibility of manufacturing larger skin panels requiring fewer joints for the assembly of the entire fuselage. The construction of such larger elements has been made possible by the development of the so-called splicing concept [3, 6]. A spliced laminate is built up by putting a metal sheet against another along a line, within the same laminate layer, called splice line. To avoid localized lack of strength, splice lines are alternated through the thickness, as shown in figure 1. Although the splicing concept represents an effective technique to build large panels, splice lines constitute discontinuities, which can lead to severe stress concentrations and delamination, if they are not carefully designed. Experimental tests and numerical analyses were carried out to investigate the composite joint behavior for different splicing geometries and metal layer thicknesses, with particular care devoted to the delamination phenomenon [15, 16]. These studies suggest that the effective and accurate prediction of the strain and stress field in proximity of the splice lines assumes a great relevance for the characterization of the mechanical behavior of different configurations. In the present paper, a twodimensional formulation for the analysis of spliced laminates with general lay-up is proposed. The problem is formulated in terms of the integral equations governing the behavior of each anisotropic layer within the laminate joint. The fundamental solutions, needed to deduce the boundary integral representation of the problem, are explicitly determined in analytical form. The laminate joint configuration as a whole is retrieved by enforcing continuity and equilibrium between contiguous layers in a multidomain approach. The formulation has been numerically solved by using the boundary element method. To ascertain the soundness of the proposed approach different configurations have been analyzed. The numerical results obtained show good agreement with analytical ones and with those obtained from the analyses carried out by using other numerical methods.

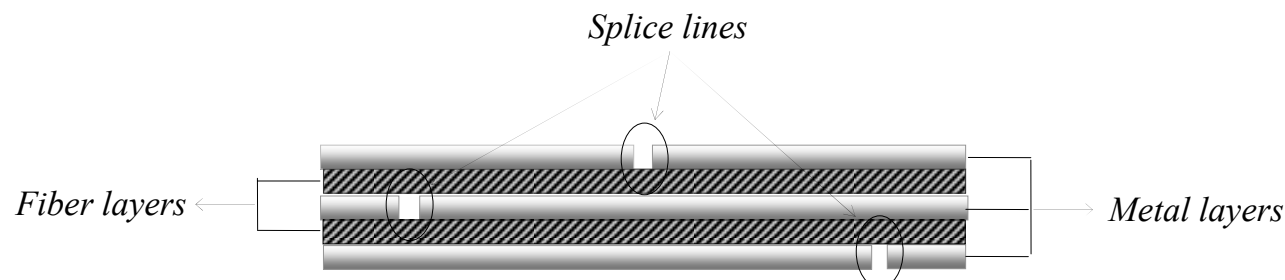

Figure 1. Spliced laminate section. 


\section{FORMULATION}

\subsection{Basic Equations}

In the two-dimensional formulation presented, the hypotheses of linear elasticity and generalized plan strain field are assumed. Let us consider the two-dimensional problems for the domain $\Omega$ lying in the $x_{1} x_{2}$ plane and bounded by the contour line $\partial \Omega$. The elastic response does not vary along the $x_{3}$ direction and the behavior of the body is described in terms of displacements $\quad \boldsymbol{u}=\left[\begin{array}{lll}u_{1} & u_{2} & u_{3}\end{array}\right]^{T}, \quad$ strains $\boldsymbol{\varepsilon}=\left[\begin{array}{llllll}\varepsilon_{11} & \varepsilon_{22} & \varepsilon_{12} & \varepsilon_{13} & \varepsilon_{23} & \varepsilon_{33}\end{array}\right]^{T}$ and stresses $\boldsymbol{\sigma}=\left[\begin{array}{llllll}\sigma_{11} & \sigma_{22} & \sigma_{12} & \sigma_{13} & \sigma_{23} & \sigma_{33}\end{array}\right]^{T}$. The strain field is linked to the mechanical displacements by the strain-displacement relations, which can be compactly written as

$$
\varepsilon=\not{D} \boldsymbol{u}
$$

where the differential operator $\mathcal{D}$ is defined as

$$
\mathscr{D}=\left[\begin{array}{cccccc}
\partial / \partial x_{1} & 0 & \partial / \partial x_{2} & 0 & 0 & 0 \\
0 & \partial / \partial x_{2} & \partial / \partial x_{1} & 0 & 0 & 0 \\
0 & 0 & 0 & \partial / \partial x_{1} & \partial / \partial x_{2} & 0
\end{array}\right]^{T}
$$

It is worthwhile to note that, in order to maintain a general and efficient matrix notation, the independence of the analysis from the $x_{3}$ direction is expressed by keeping $\partial / \partial x_{3}=0$, while also the zero component $\varepsilon_{33}$ of the strain field is kept in the formulation. This allows flexibility in the formulation development and in its implementation in computer codes. The equilibrium equations are

$$
\mathfrak{D}^{T} \boldsymbol{\sigma}+\boldsymbol{f}=\boldsymbol{0}
$$

where $\boldsymbol{f}=\left[\begin{array}{lll}f_{1} & f_{2} & f_{3}\end{array}\right]^{T}$ is the body force vector. Finally, the constitutive equations can be expressed by

$$
\boldsymbol{\sigma}=\boldsymbol{E} \varepsilon
$$

where $\boldsymbol{E}$ is the elasticity matrix. By combining Eqs. (1), (3) and (4) the governing equations of the problem can be expressed in the compact form

$$
\mathfrak{D}^{T} \boldsymbol{E} \mathcal{D} \boldsymbol{u}+\boldsymbol{f}=\mathbf{0}
$$

The essential and natural boundary conditions associated with Eq. (5) can be expressed in the form

$$
\begin{array}{cr}
\boldsymbol{u}=\overline{\boldsymbol{u}} & \text { on } \partial \Omega_{1} \\
\boldsymbol{t}=\mathcal{D}_{n}{ }^{T} \boldsymbol{E} \mathcal{D} \boldsymbol{u}=\overline{\boldsymbol{t}} & \text { on } \partial \Omega_{2}
\end{array}
$$


where $\boldsymbol{t}=\left[\begin{array}{lll}t_{1} & t_{2} & t_{3}\end{array}\right]^{T}$ is the vector of the boundary tractions and $\partial \Omega_{1}$ and $\partial \Omega_{2}$ represent the constrained and loaded portions of the boundary, respectively. $\mathbb{D}_{n}$ is the boundary traction operator, obtained by substituting in the operator $\mathscr{D}$ the derivatives with the corresponding boundary outer normal direction cosines.

\subsection{Boundary integral representation}

Let $\boldsymbol{u}_{j}$ be a solution of the elastic problem satisfying the equilibrium equations for a system of body forces $\boldsymbol{f}_{j}$ so that

$$
\mathscr{D}^{T} \boldsymbol{E} D \boldsymbol{u}_{j}+\boldsymbol{f}_{j}=\mathbf{0}
$$

Let $\boldsymbol{\sigma}_{j}$ and $\boldsymbol{\varepsilon}_{j}$ denote the stress and strain fields associated with $\boldsymbol{u}_{j}$, and let $\boldsymbol{t}_{j}$ be the corresponding boundary tractions. By using this solution and the actual elastic solution, one writes the following reciprocity statement $[17,18]$

$$
\int_{\partial \Omega}\left(\boldsymbol{u}_{j}^{T} \boldsymbol{t}-\boldsymbol{t}_{j}^{T} \boldsymbol{u}\right) d \partial \Omega=\int_{\Omega}\left(\boldsymbol{f}_{j}^{T} \boldsymbol{u}-\boldsymbol{u}_{j}^{T} \boldsymbol{f}\right) d \Omega
$$

Thus, the boundary integral representation is obtained from Eq. (8) by considering a particular displacement field $\boldsymbol{u}_{j}$ corresponding to a concentrated force $\boldsymbol{f}_{j}$ acting in an infinite domain and applied at the point $P_{0}$. It is expressed by

$$
\boldsymbol{f}_{j}=\boldsymbol{c}_{j} \delta\left(P-P_{0}\right)
$$

where $\boldsymbol{c}_{j}$ is the load intensity and $\delta\left(P-P_{0}\right)$ is the Dirac's function. By using three independent particular solutions obtained for concentrated loads directed along the reference axes $(j=1,2,3)$, known as fundamental solutions, the reciprocity theorem, i.e. Eq. (8), leads to the following boundary integral representation of the displacement field [17].

$$
\boldsymbol{c}^{*} \boldsymbol{u}\left(P_{0}\right)+\int_{\partial \Omega}\left(\boldsymbol{t}^{*} \boldsymbol{u}-\boldsymbol{u}^{*} \boldsymbol{t}\right) d \partial \Omega=\int_{\Omega} \boldsymbol{u}^{*} \boldsymbol{f} d \Omega
$$

Eq. (10) represents the Somigliana identity for elasticity expressed in matrix notation. It allows to express the three displacement components at the point $P_{0}$ as function of the displacements and tractions on the boundary of the body. The kernels $\boldsymbol{u}^{*}$ and $\boldsymbol{t}^{*}$ are defined as $\boldsymbol{u}^{*}=\left[u_{i j}\right]^{T}$ and $\boldsymbol{t}^{*}=\left[t_{i j}\right]^{T}$, where $u_{i j}$ and $t_{i j}$ are the i-th component of the displacements and tractions of the j-th fundamental solution. The matrix $c^{*}$ can be calculated according to the following relationship [19]

$$
c^{*}=-\int_{\partial \Omega} t^{*} d \partial \Omega
$$

It is worth noting that the matrix $c^{*}$, calculated by Eq. (11), is related to the 
fundamental solution load intensity $\boldsymbol{c}_{j}$ (see section 2.3) and its value depends on the local geometry near the point $P_{0}[17,18]$. If the point $P_{0}$ belongs to the boundary $\partial \Omega$, Eq. (10) involves boundary quantities only and it becomes the integral equation providing the link between the variables on the boundary. This integral equation, coupled with the appropriate boundary conditions, allows the determination of the unknown variables on the boundary. Once these are determined, the displacement at each internal point $P_{0}$ can be calculated by means of Eq. (10). The strains are obtained in a pointwise fashion by appropriately differentiating Eq. (10) with respect to the source point $P_{0}$ and the following boundary integral representation for the strain field holds [18]

$$
\varepsilon\left(P_{0}\right)=\int_{\partial \Omega}\left(\boldsymbol{\Xi}^{*} \boldsymbol{t}-\boldsymbol{\Theta}^{*} \boldsymbol{u}\right) d \partial \Omega+\int_{\Omega} \boldsymbol{\Xi}^{*} \boldsymbol{f} d \Omega
$$

where

$$
\begin{aligned}
& \boldsymbol{\Theta}^{*}=-\not D c^{*-1} \boldsymbol{t}^{*} \\
& \boldsymbol{\Xi}^{*}=-\not D c^{*-1} \boldsymbol{u}^{*}
\end{aligned}
$$

Finally, the boundary integral representation for the stresses is simply obtained through the constitutive equations and one has

$$
\boldsymbol{\sigma}\left(P_{0}\right)=\boldsymbol{E} \boldsymbol{\varepsilon}\left(P_{0}\right)=\int_{\partial \Omega}\left(\boldsymbol{E} \boldsymbol{\Xi}^{*} \boldsymbol{t}-\boldsymbol{E} \boldsymbol{\Theta}^{*} \boldsymbol{u}\right) d \partial \Omega+\int_{\Omega} \boldsymbol{E} \boldsymbol{\Xi}^{*} \boldsymbol{f} d \Omega
$$

\subsection{Fundamental solutions}

The boundary integral representation relies on the knowledge of the displacement field $\boldsymbol{u}_{j}$ corresponding to a concentrated point force acting at a point in the infinite domain. It is the fundamental solution governed by the following equation

$$
D^{T} \boldsymbol{E} D \boldsymbol{u}_{\boldsymbol{j}}+\boldsymbol{c}_{j} \delta\left(P-P_{0}\right)=\mathbf{0}
$$

Starting from the original work of Eshelby et al. [20] and Stroh [21] solutions of Eq. (16) have been proposed by many authors for anisotropy [22-24] and piezoelasticity $[25,26]$. The fundamental solution employed in this paper is obtained by a modified Lekhnitskii's approach proposed by Davì and Milazzo [27-29]. Eq. (16) suggests for the particular solution the following form

$$
\boldsymbol{u}=\lambda \boldsymbol{a} \ln \left(X_{1}+\mu X_{2}\right)
$$

where $\boldsymbol{a}, \mu$ and $\lambda$ are complex constants to be determined and

$$
X_{i}=x_{i}(P)-x_{i}\left(P_{0}\right) \quad(i=1,2)
$$

Substitution of Eq. (17) into Eq. (16) leads to the eigenvalue problem

$$
\left[\boldsymbol{I}_{1}^{T} \boldsymbol{E I}_{1}+\mu\left(\boldsymbol{I}_{1}^{T} \boldsymbol{E} \boldsymbol{I}_{2}+\boldsymbol{I}_{2}^{T} \boldsymbol{E I}_{1}\right)+\mu^{2} \boldsymbol{I}_{2}^{T} \boldsymbol{E} \boldsymbol{I}_{2}\right] \boldsymbol{a}=\mathbf{0}
$$


where the matrix $\boldsymbol{I}_{m} \quad(m=1,2)$ is obtained from the operator $\mathscr{D}$ by setting the derivatives with respect to $x_{m}$ equal to one and replacing all the other terms with zeros. The solution of Eq. (19) gives six eigenvalues $\mu_{k}$ and the corresponding eigenvectors $\boldsymbol{a}_{k}$, which form conjugate pairs for stable materials. The fundamental solutions are then obtained by superposing the solutions of the form (17) associated with the six eigenvalues $\mu_{k}$. By choosing the eigenvalues such that $\operatorname{Im}\left(\mu_{k}\right)>0$, the displacements $\boldsymbol{u}_{j}$ of the fundamental solutions can be expressed as

$$
\boldsymbol{u}_{j}=2 \sum_{k=1}^{3} \operatorname{Re}\left[\lambda_{k j} \boldsymbol{a}_{k} \ln \left(X_{1}+\mu_{k} X_{2}\right)\right]
$$

The generalized tractions are obtained from the following relation

$$
\boldsymbol{t}_{j}=2 \sum_{k=1}^{3} \operatorname{Re}\left[\lambda_{k j} \mathscr{D}_{n}^{T} \boldsymbol{E} \mathcal{D}_{\mu_{k}} \boldsymbol{a}_{k} \frac{1}{X_{1}+\mu_{k} X_{2}}\right]
$$

where the matrix $\mathscr{D}_{\mu_{k}}$ is obtained from the operator $\not{D}$ by replacing the derivatives with respect to $x_{1}$ with one and the derivatives with respect to $x_{2}$ with $\mu_{k}$. The constants $\lambda_{k j}$ are determined by enforcing the compatibility and equilibrium conditions on the complex Gauss plane [29]. By so doing the vector $\lambda_{j}=\left[\begin{array}{lll}\lambda_{1 j} & \lambda_{2 j} & \lambda_{3 j}\end{array}\right]^{T}$ is obtained from

$$
\lambda_{j}=\left(\boldsymbol{B}+\tilde{\boldsymbol{B}} \tilde{\boldsymbol{A}}^{-1} \boldsymbol{A}\right)^{-1} \boldsymbol{c}_{j}
$$

In Eq. (22), the tilde denotes the complex conjugate, $\mathbf{A}$ is the eigenvectors matrix and the columns $\boldsymbol{b}_{k}$ of the matrix $\mathbf{B}$ are defined as

$$
\boldsymbol{b}_{k}=\overline{\mathscr{D}}_{k} \boldsymbol{E} \mathscr{D}_{\mu k} \boldsymbol{a}_{k}
$$

where

$$
\overline{\boldsymbol{D}}_{k}=\pi \frac{1+\sqrt{-1} \mu_{k}}{1+\mu_{k}^{2}}\left[\begin{array}{cccccc}
-1 & 0 & 0 & 0 & 0 & \sqrt{-1} \\
0 & \sqrt{-1} & 0 & 0 & 0 & -1 \\
0 & 0 & 0 & \sqrt{-1} & -1 & 0
\end{array}\right]
$$

It is worth to note that in the calculation of the present fundamental solution a suitable matrix notation has been adopted, which proved to be very effective for computer implementation purposes.

\subsection{Numerical model and solution}

The boundary integral formulation is solved numerically by the boundary element method. The boundary $\partial \Omega$ is discretized into $m$ boundary elements and over each one 
of these elements $\partial \Omega_{(k)}$ the displacements $\boldsymbol{u}$ and the tractions $\boldsymbol{t}$ are expressed by means of their respective nodal values $\boldsymbol{\Delta}_{(k)}$ and $\boldsymbol{P}_{(k)}$

$$
\begin{array}{ll}
\boldsymbol{u}=\boldsymbol{N} \boldsymbol{\Delta}_{(k)} & \text { on } \partial \boldsymbol{\Omega}_{(k)} \\
\boldsymbol{t}=\boldsymbol{\Psi} \boldsymbol{P}_{(k)} & \text { on } \partial \boldsymbol{\Omega}_{(k)}
\end{array}
$$

where $\boldsymbol{N}$ and $\boldsymbol{\Psi}$ are matrices of standard shape functions whose order depends on the class of boundary elements used in the numerical model. In the absence of body forces, the discretized version of Eq. (10) for the point $P_{i}$ is therefore given by

$$
\boldsymbol{c}_{i}^{*} \boldsymbol{u}\left(P_{i}\right)+\sum_{k=1}^{m} \boldsymbol{H}_{i k} \boldsymbol{\Delta}_{(k)}+\sum_{k=1}^{m} \boldsymbol{G}_{i k} \boldsymbol{P}_{(k)}=0
$$

where

$$
\begin{aligned}
\boldsymbol{H}_{i k} & =\int_{\partial \Omega_{(k)}} \boldsymbol{t}^{*}\left(P, P_{i}\right) \boldsymbol{N}(P) d \partial \Omega \\
\boldsymbol{G}_{i k} & =-\int_{\partial \Omega_{(k)}} \boldsymbol{u}^{*}\left(P, P_{i}\right) \boldsymbol{\Psi}(P) d \partial \Omega
\end{aligned}
$$

By taking the point $P_{i}$ to all boundary nodes using a collocation technique and absorbing the $\boldsymbol{c}_{i}^{*}$ matrix with the corresponding block of $\boldsymbol{H}_{i i}$, a linear algebraic system is obtained. It can be compactly written as [29]

$$
H \Delta-G P=0
$$

where $\boldsymbol{H}$ and $\boldsymbol{G}$ are the square influence matrices, obtained assembling the $\boldsymbol{H}_{i k}$ and $\boldsymbol{G}_{i k}$ blocks; $\boldsymbol{A}$ is the vector containing the nodal values of the displacements and $\boldsymbol{P}$ is the vector of the nodal values of the tractions. Eq. (30), coupled to the boundary conditions given in terms of prescribed nodal values, provides the solution of the problem.

\subsection{Multidomain technique}

When the investigated domain is made up of piece-wise different materials or when cracks and/or inclusions are present, the problem can be solved by using a multidomain approach [28-32]. This BEM technique is based on the splitting up of the original domain into homogeneous subregions. These are discretized by boundary elements with common interfaces sharing the same nodes, so that Eq. (30) still holds for each single subdomain. The following discretized integral equations can be written, one for each subregion

$$
\boldsymbol{H}^{(i)} \boldsymbol{\Delta}^{(i)}-\boldsymbol{G}^{(i)} \boldsymbol{P}^{(i)}=\mathbf{0} \quad(i=1,2 \ldots . M)
$$

where $M$ is the number of subregions considered and the superscript $(i)$ denotes quantities associated with the $i$-th subdomain. To obtain the final numerical model, the domain integrity need to be restored by enforcing the displacement continuity and traction equilibrium conditions along the interfaces between contiguous subdomains. 
Let us introduce a partition of the linear algebraic system given by Equation (31) in such a way that the generic vector $\boldsymbol{y}^{(i)}$ can be written as

$$
\boldsymbol{y}^{(i)}=\left[\begin{array}{c}
\boldsymbol{y}_{\partial \Omega_{\Omega_{11}}^{(i)}} \\
\vdots \\
\boldsymbol{y}_{\partial \Omega_{\alpha_{M}}}^{(i)}
\end{array}\right]
$$

where the vector $\boldsymbol{y}_{\partial \Omega_{i j}}^{(i)}$ collects the components of $\boldsymbol{y}^{(i)}$ associated with the nodes belonging to the interface $\partial \Omega_{i j}$ between the $i$-th and $j$-th subdomain. The convention that $\partial \Omega_{i i}$ denotes the external boundary of the $i$-th sub domain is assumed (see Figure 2). By so doing, in the discretized model the interface compatibility and equilibrium conditions, that is the interface continuity conditions, are written as

$$
\begin{array}{cc}
\boldsymbol{\Delta}_{\partial \Omega_{i j}}^{(i)}=\boldsymbol{\Delta}_{\partial \Omega_{i j}}^{(j)} & (i=1 \ldots . . M-1 ; j=i+1 \ldots M) \\
\boldsymbol{P}_{\partial \Omega_{i j}}^{(i)}=-\boldsymbol{P}_{\partial \Omega_{i j}}^{(j)} & (i=1 \ldots . M-1 ; j=i+1 \ldots M)
\end{array}
$$

If the $i$-th and $j$-th subdomain have no common boundary, $\boldsymbol{y}_{\partial \Omega_{i j}}^{(i)}$ is a zero-order vector and Eqs. (33) and (34) are no longer valid. The Eqs. (31), (33) and (34) provides an algebraic system that, together with the boundary conditions prescribed on the external boundaries $\partial \Omega_{i i}(i=1 \ldots \mathrm{M})$, allows the determination of the mechanical response in terms of nodal displacements and tractions on the boundary of each subdomain.

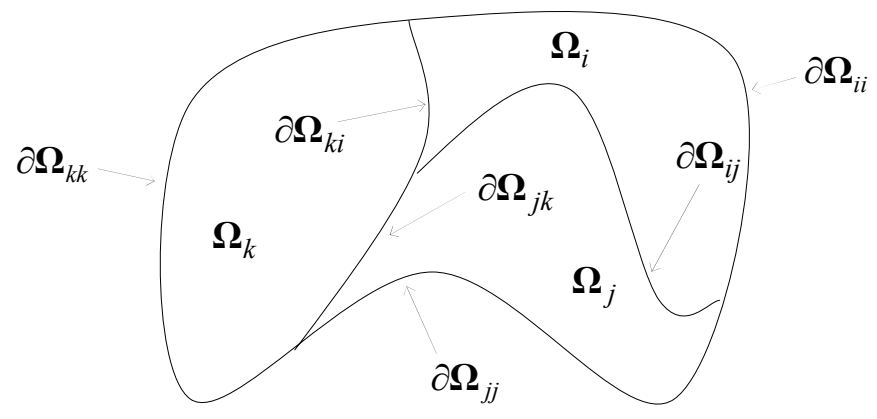

Figure 2. Multidomain configuration.

\section{COMPUTER CODE}

The formulation presented has been implemented in a numerical code, called BEMJNT, by using MATLAB ${ }^{\circledR}$ and $\mathrm{C}^{++}$routines. The code is arranged in a structure consisting of three main blocks, shown in Figure 3, which address pre-processing, solution and post-processing tasks respectively. The PREPROC block provides the elaboration of the input data, implementing geometry and discretization. In this phase 


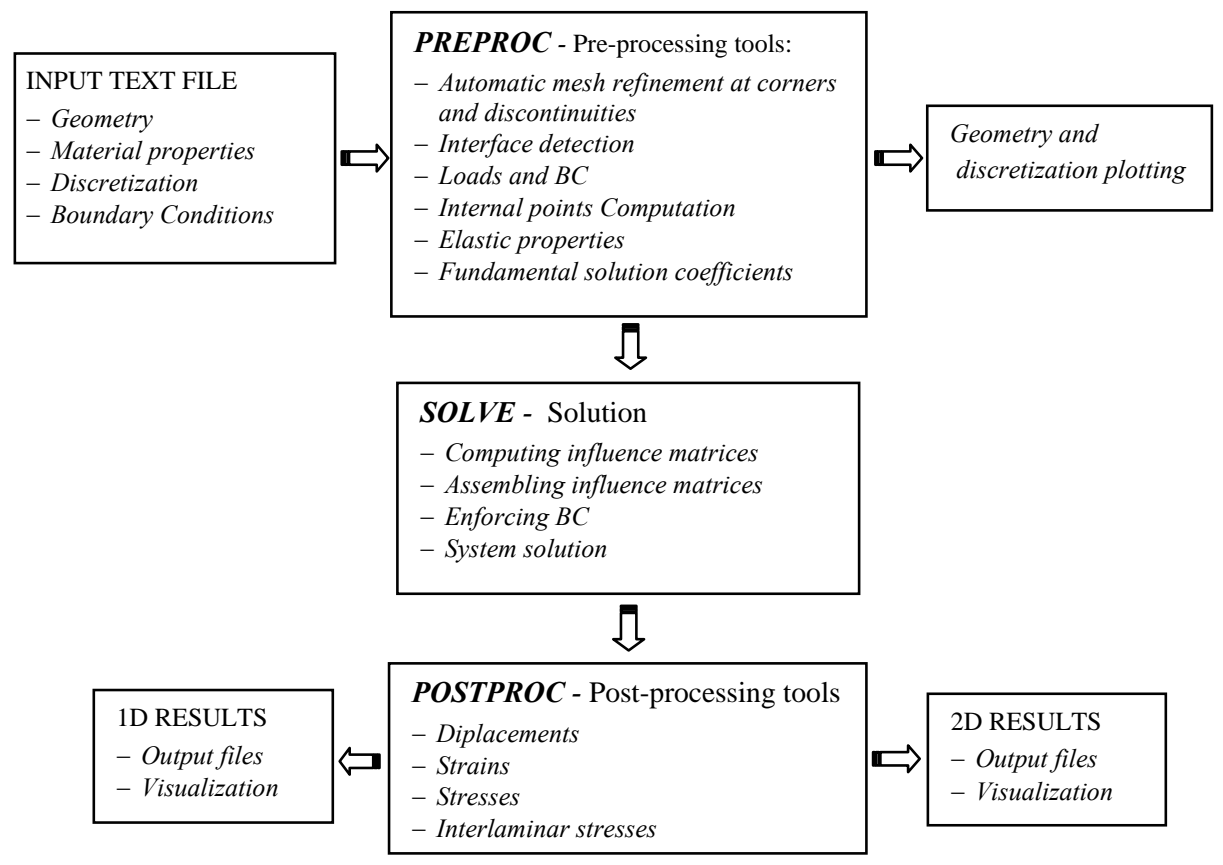

Figure 3. Structure of the computer code BEM-JNT.

the fundamental solutions coefficients are also computed by a dedicated subroutine. The input data, consisting of geometry, material properties, boundary conditions and discretization parameters, are defined in a text file, which allow simple and flexible definition of alternative parametric configurations. The developed pre-processing tools are well suited to the class of structural problems considered like composite joints. Indeed, in the context of the multidomain approach, they make possible rapid modeling of laminated structures with general lay-up, with or without splice lines and cracks. Computation with constant and linear boundary elements for both displacements and tractions interpolation is allowed. It is worth noting that each sub-region is modeled independently with the only requirement that the boundaries defining the interfaces of contiguous domain have the same discretization. Moreover, the discretization routine automatically fits the mesh when corners are approached in order to catch stress and tractions concentrations occurring near the boundary layer. In particular, a logarithmically spaced refinement is automatically introduced, for a length based upon discretization parameters, when a corner is detected by means of a suitable check of the normals of contiguous input contours. The block SOLVE accomplishes the computation of the influence matrices defined in Eq. (30). The influence coefficients of the matrices $\boldsymbol{H}$ and $\boldsymbol{G}$ and the coefficients of the matrix $\mathbf{c}^{*}$ are computed by an integration scheme based on Gaussian quadrature, which takes the kernel singularities into account through the subtraction of singularity method and employs an automatic element subdivision approach for the computation of near singular integrals [17]. To enforce the interface continuity conditions and restore the structure integrity, an algorithm for the detection of the common nodes at the interface between two contiguous sub-domains has been 
implemented. Once the common nodes are detected, the SOLVE routine provides the assembling of the influence matrices and the enforcing of the boundary conditions. The solution of the algebraic system is then found by means of standard routines. Postprocessing tools, implemented in the block POSTPROC, provide complete information about the two-dimensional stress, strain and displacement distribution and interlaminar tractions prediction. The inherent features of the boundary formulation developed, the use of effective compact matrix notation as well as the code implementation strategy provide a powerful tool for analysis of laminated joint structures in the framework of the discussed applications.

\section{APPLICATIONS AND DISCUSSIONS}

The computer code developed has been used to perform some computations with the aim to validate the method and then to analyze the behavior of typical configurations employed in Glare ${ }^{\circledR}$ joints.

\subsection{Validation analysis}

To validate the proposed approach the analysis of an aluminum sheet bonded to a rigid body by an adhesive layer has been performed. The geometry, the boundary conditions and the material properties are shown in Figure 4. This simple analysis has been carried out to validate the formulation as well as the numerical code. The present results have been compared with those obtained by solving the corresponding one-dimensional analytical model [33]. Indeed, one-dimensional modeling based on the classical laminates theory is often used to get a general idea of the overall stress distribution within the laminate avoiding time-consuming procedures based on more complex formulations. Nevertheless, the detail level obtained from such analysis can be unsatisfactory for more refined applications. From this point of view, the proposed model is capable of completely assessing the distribution of internal stresses as well as interlaminar tractions in the analyzed laminate joint. Additionally, the present results have been compared with those obtained by the finite element code MSC.Nastran ${ }^{\mathrm{TM}}$. This allowed to point out the reduction of the analysis time and of the required computational resources connected to the boundary integral equation approach. Figure 5 shows the results obtained for the one-dimensional generalized displacements of the aluminum ply, namely $u_{1}\left(x_{1}\right), u_{2}\left(x_{1}\right)$ and the rotation $\varphi\left(x_{1}\right)$ [33], suitably arranged by postprocessing the computed displacement field. These results are compared with those

$$
\begin{aligned}
& E_{\mathrm{Al}}=72.5 G p a \\
& v_{\mathrm{Al}}=0.3 \\
& G_{\mathrm{Ad}}=0.64 \mathrm{Gpa} \\
& v_{\mathrm{Al}}=0.3
\end{aligned}
$$

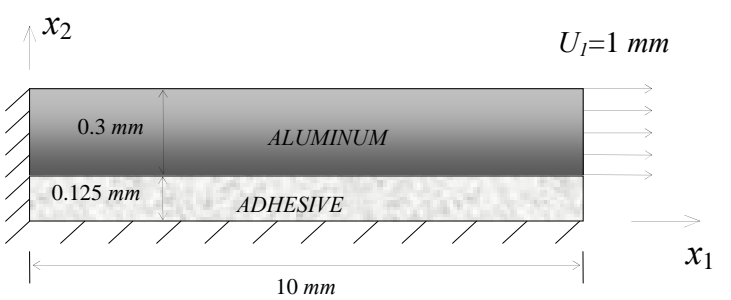

Figure 4. Geometry and boundary conditions for the aluminum sheet bonded to a rigid body by an adhesive layer. 


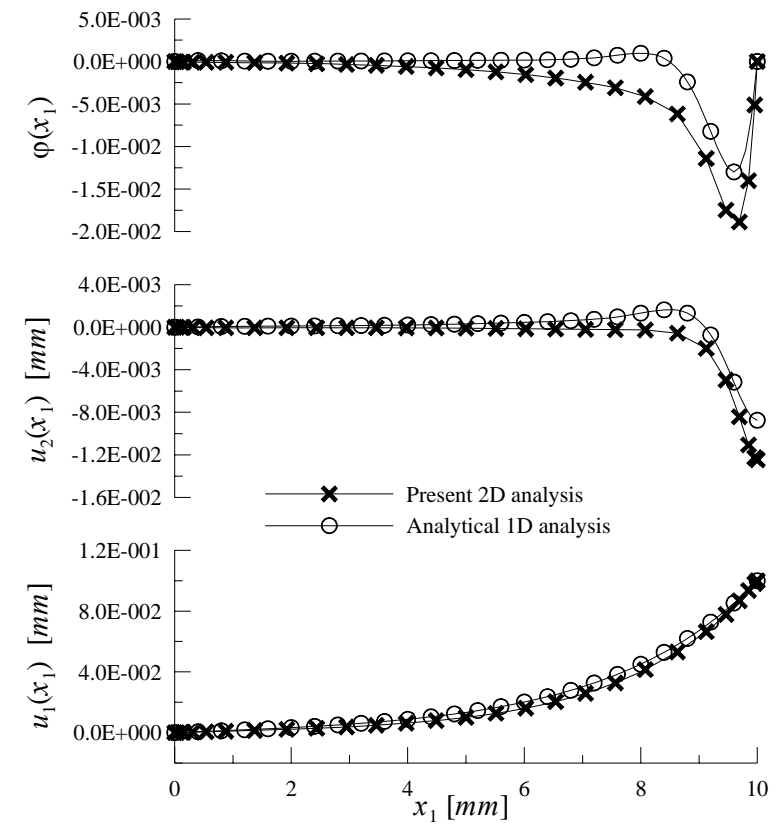

Figure 5. 1D generalized displacement distributions for the aluminum sheet bonded to a rigid body by an adhesive layer.
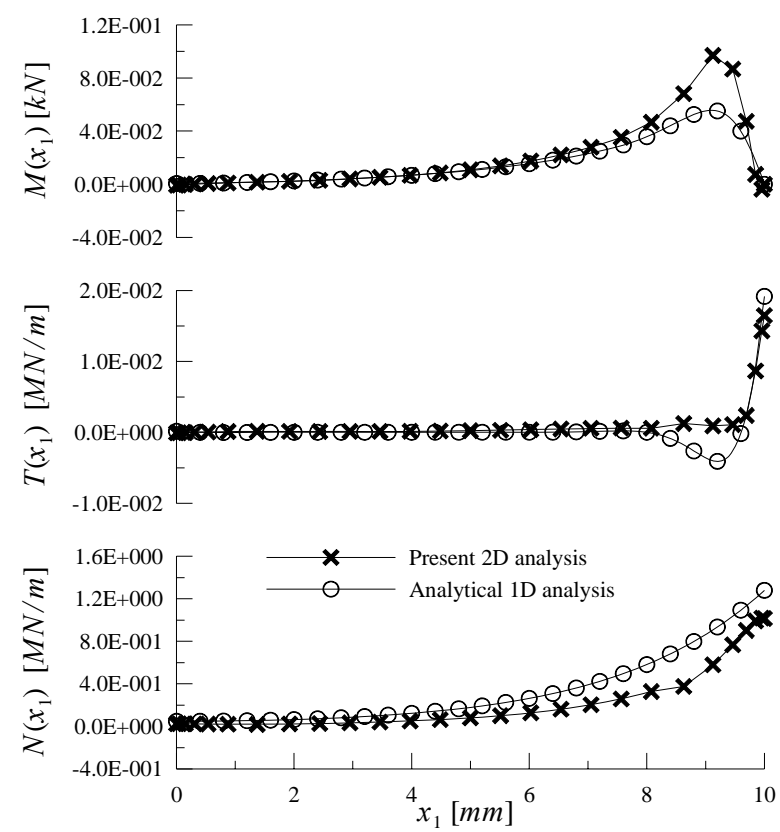

Figure 6. Stress resultant distributions for the aluminum sheet bonded to a rigid body by an adhesive layer. 

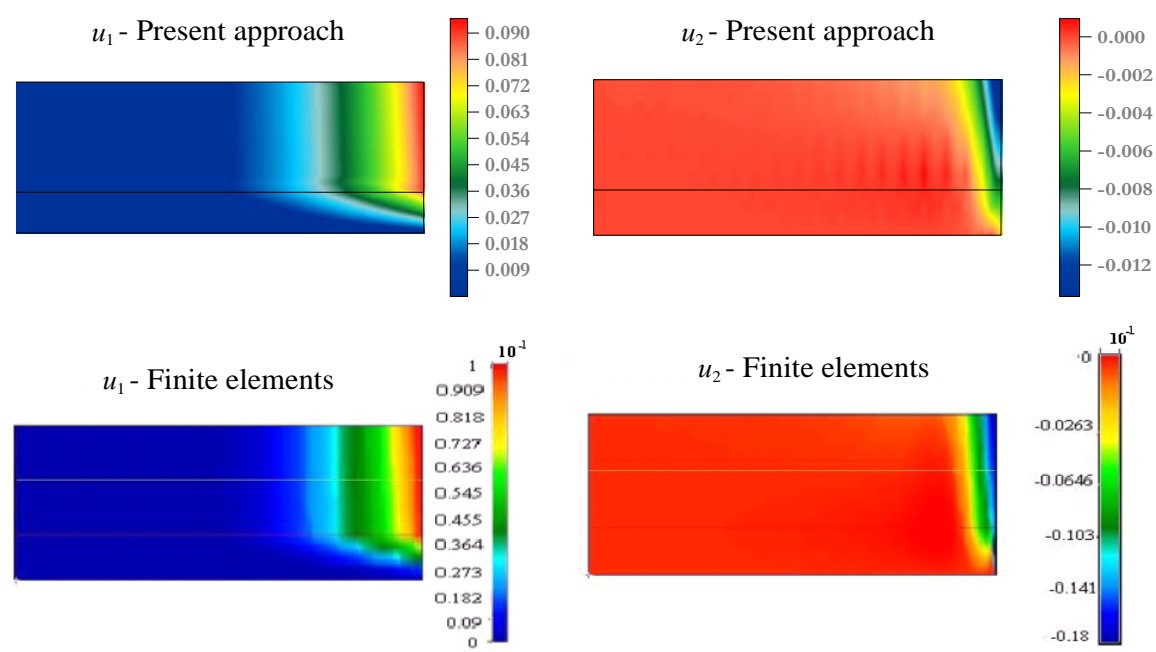

Figure 7. Displacement distributions for the aluminum sheet bonded to a rigid body by an adhesive layer: a) present approach; b) finite elements.

obtained from one-dimensional analysis and generally, good agreement is achieved. Some discrepancy occurs between one-dimensional and present results close to the free edge of the adhesive ply where the highest differences between the $1 \mathrm{D}$ analysis and the 2D boundary element analysis are observable. This circumstance is primarily a consequence of the effect of the transverse contraction of the adhesive ply that is particularly strong close to the free edge and that is not taken into account in 1D model. Analogous considerations can be done about the normal stress, shear stress and bending moment resultant distributions, which are shown in Figure 6. To validate the analysis and point out the features of the proposed approach a comparison of the present results with those obtained by using finite elements has been carried out. Figures 7.a show the distribution of the joint displacement components computed by the present method by using a discretization with 120 constant boundary elements and 300 internal points. Figures 7.b show the distribution of the joint displacement components computed by the finite element code MSC.Nastran ${ }^{\text {TM }}$ by a mesh of 1700 CQUAD4 elements with 1818 nodes. The results of the two analyses in terms of displacements match well to each other. This circumstance is also true for the stress distributions, which are not provided for brevity. The analysis evidences the soundness of the proposed approach, which provides accurate results with reduced computational resources due to the dimensionality reduction inherent to the boundary element approach.

\subsection{Glare ${ }^{\circledR}$ laminate with splice lines}

The second application deals with the typical configuration for a Glare ${ }^{\circledR}$ laminate built with the splice line joint technique, which is shown in Figure 8. The material used is Glare $^{\circledR}$ 3/2 0.3 with Al 2024-T3 metallic plies and $0.125 \mathrm{~mm}$ thick [0/90] S2 Glass/Epoxy plies. The laminate is clamped on the left side and a constant displacement 


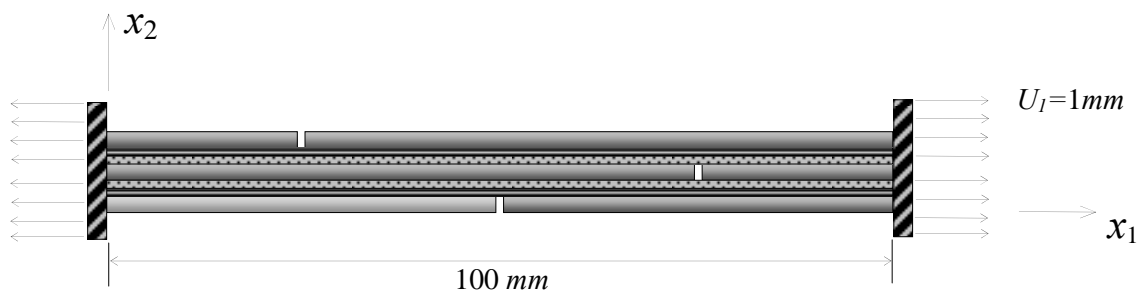

Figure 8. Glare® laminate with splice lines: geometry and boundary conditions.

distribution is imposed on the opposite side along the $x_{1}$ axis as shown in Figure 8. The splice lines are modeled by imposing traction free conditions between the contiguous domains belonging to the considered spliced layer. Nevertheless, adhesive filled splice lines can be modeled by virtue of the multidomain implementation by simply adding a subregion with the adhesive properties. The analysis was performed with 2400 constant boundary elements and the results were compared with those obtained by finite elements. For both displacements and stresses good agreement was obtained between present and finite element results, which are not presented for the sake of brevity. Figure 9 and 10 show the distributions of $\sigma_{11}$ and the interlaminar stresses along the aluminum-composite interfaces, respectively, which are representative of the laminate behavior. Stress concentrations can be observed at the tips of the splice lines and it is worth noting that the effects of the discontinuities introduced by the splice lines even affect the unspliced plies. This realistic application point out the potentiality of the proposed formulation, which is able to capture stress concentrations and singular behavior without requiring very fine meshes.

\subsection{Glare ${ }^{\circledR}$ laminate with an external doubler.}

This application deals with a Glare ${ }^{\circledR}$ laminate with an external doubler under uniform extension. This analysis has been carried out with the aim to show the ply drop-off effect [33] induced by the presence of the doubler. The geometry and the boundary conditions are shown in Figure 11 while the material used as well as the stacking sequence and the thicknesses of the laminae are the same as the previous example. The doubler is modeled by an aluminum ply having thickness $0.3 \mathrm{~mm}$. Due to the symmetry of the joint, only one-half of the structure has been modeled with eight different plies. To model the splice line, free edge boundary conditions are imposed. The distribution of the stress $\sigma_{11}$ in the proximity of the free edge of the doubler is shown in Figure 12, whereas Figure 13 depicts the interlaminar stresses along the aluminum-composite interfaces. Again, the present approach is able to catch efficiently the stress concentration due to the thickness discontinuity induced by the presence of the doubler. Considering that the present results have been obtained with a discretization of 1266 constant boundary elements, the considerations on the approach effectiveness quoted for the previous example are confirmed. 

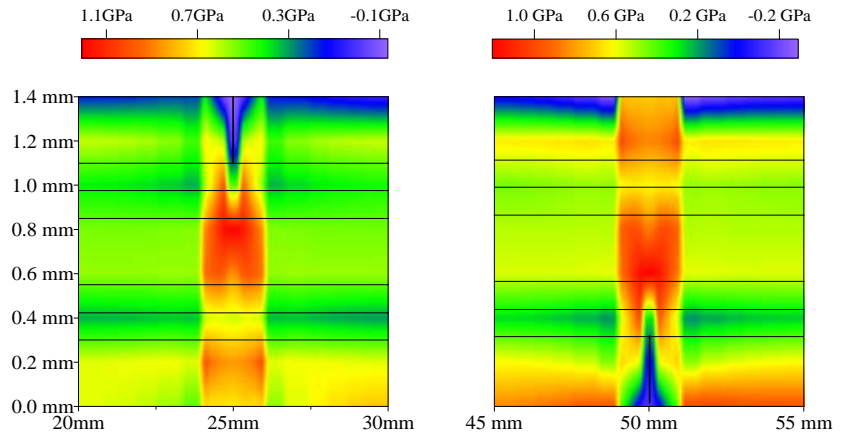

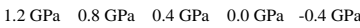

Figure 9. $\sigma_{11}$ distribution for the Glare ${ }^{\circledR}$ laminate with splice lines.
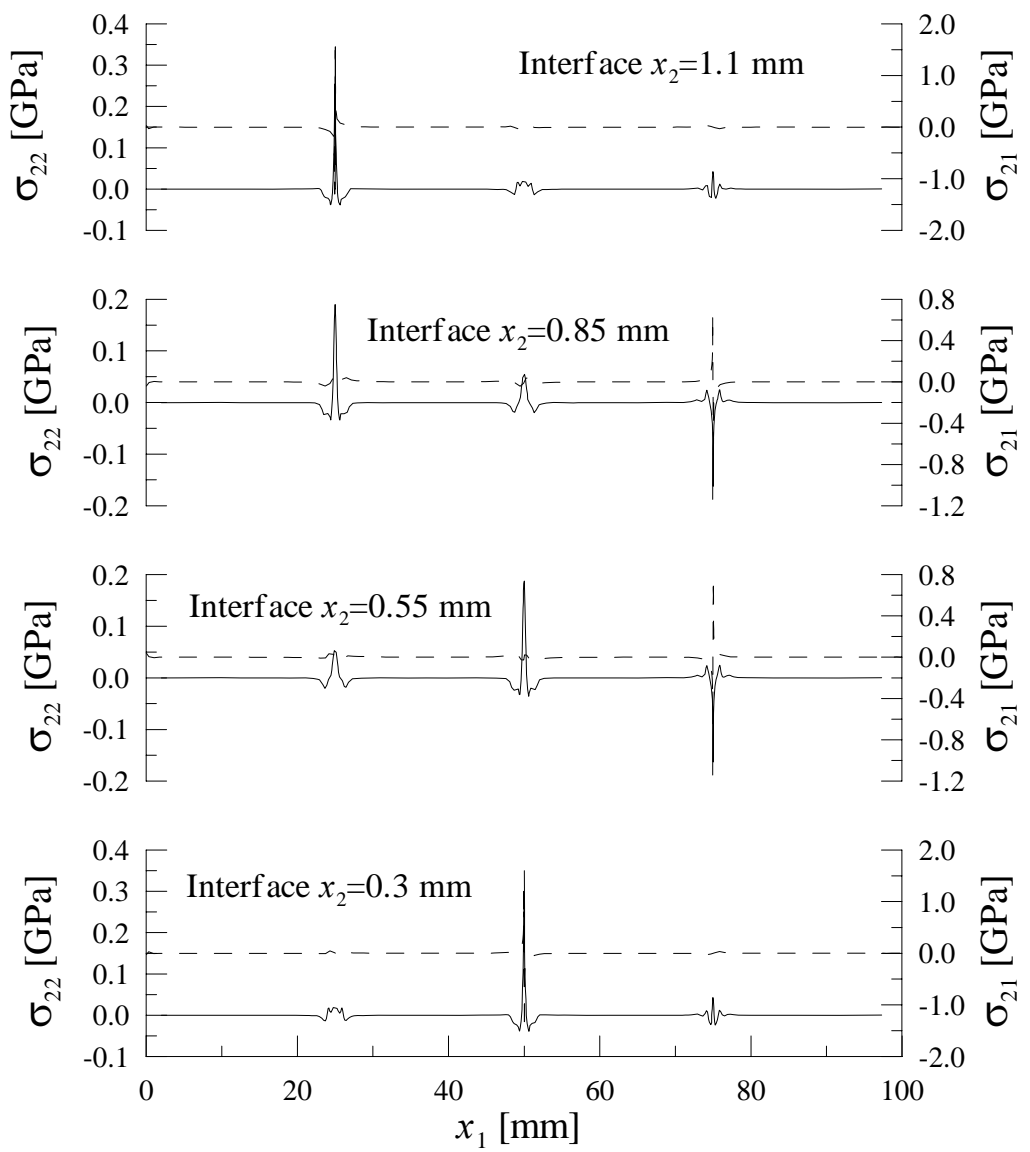

Figure 10. Interlaminar stress distribution for the Glare ${ }^{\circledR}$ laminate with splice lines.

$$
---\sigma_{21} ; \sigma_{22}
$$




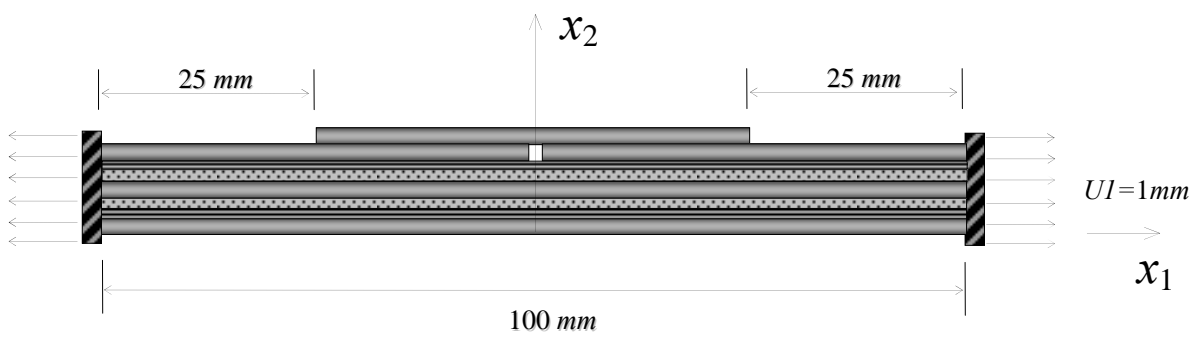

Figure 11. Glare ${ }^{\circledR}$ laminate with external doubler: geometry and boundary conditions.

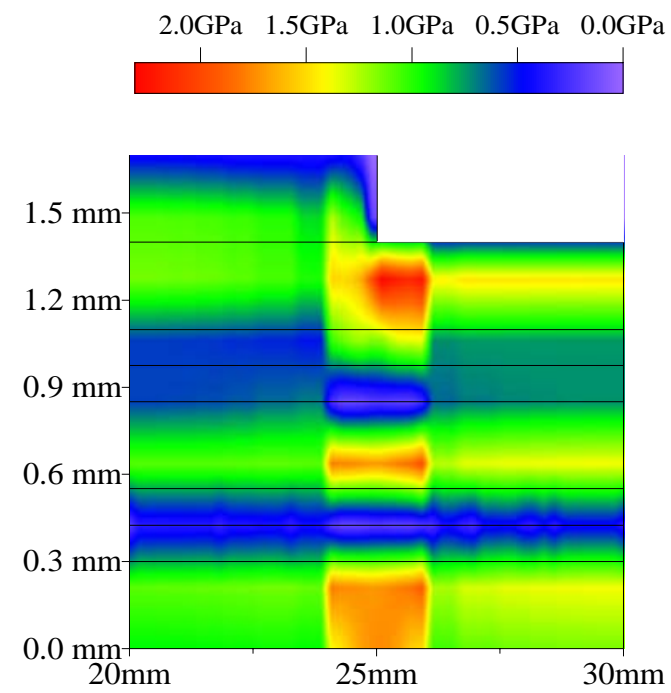

Figure 12. $\sigma_{11}$ distribution for the Glare ${ }^{\circledR}$ laminate with external doubler.

\section{CONCLUSIONS}

The paper concerns boundary element modeling and analysis of adhesive bonded laminate joints. A multidomain boundary element formulation has been presented for the analysis in the framework of two-dimensional anisotropic elasticity and attention has been focused on the application to Glare ${ }^{\circledR}$ laminate and joints built with the spliced concept technique. The formulation allows the analysis of general lay-up laminates and it can be effectively used to study the effects of the splice lines on the overall stress distribution as well as on the interlaminar stresses. Numerical results have shown good agreement with the results obtained by other numerical methods. The capability of pointing out stress concentrations, as well as the interlaminar tractions in the proximity of the splice tips, make the approach presented a powerful tool to characterize the structural suitability of the configurations analyzed. Moreover, the computational efforts reduction, attained by the boundary element method, associated with the use of simple 
parametrical input to define geometry and mesh, lead to an overall time reduction needed for the analysis. From this point of view the present approach, which considers the perfectly bonded interface, could be extended to imperfect bonding conditions e.g. linear models for imperfect bonding between two adjacent layers. This requires the employment of suitable interface relationships between displacements and tractions, e.g. those involved in linear models for imperfect bonding between two adjacent layers. This development of the method is in progress and it will be subject of future papers.
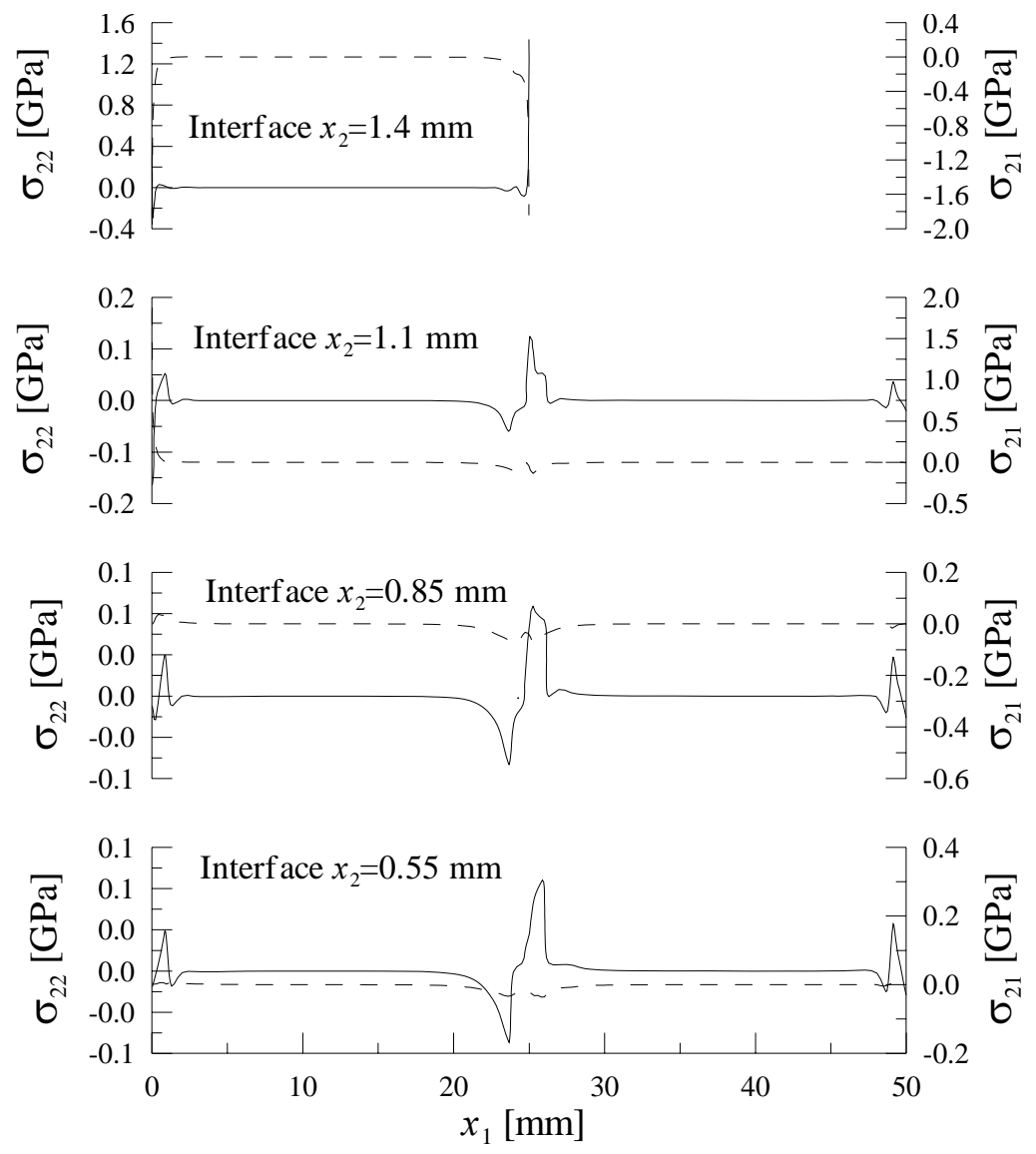

Figure 13. Interlaminar stresses for the Glare ${ }^{\circledR}$ laminate with external doubler.

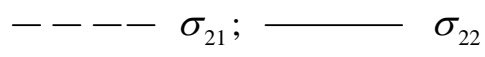

\section{REFERENCES}

1. Noor A.K., Venneri S.L., Paul D.B., Hopkins M.A. Structure technology for future aerospace systems. Computers and Structures, Vol. 74: pp. 507-519, 2000.

2. Hinrichsen J., Bautista C.. The Challenge of Reducing both Airframe Weight and Manufacturing Cost. Air \& Space Europe, Vol. 3 : pp. 119-121, 2001. 
3. Roebroeks G.H.J.J. Fiber Metal Laminates, Recent Development and Application. Symp. On Fatigue of Aircraft Material, Delft , 1992.

4. Gunnink J.W., Vlot A., de Vries T.J., Van Der Hoeven W. Glare Technology Development 1997-2000. Applied Composite Materials, Vol. 9:pp 201-219, 2002.

5. Vogelesang LB, Marissen R, Schijve J. A new fatigue resistant material: aramid reinforced aluminum laminate (ARALL). In: Proceedings of the 11th ICAF Symposium, Noordwijkerhout- NLR, pp. 3.4/1-.4/39, 1991.

6. Asundi A., Alta Choi T.J.. Fiber Metal Laminates: An Advanced Material for Future Aircraft. Journal of Material Processing Technology, Vol. 63: pp.384-394, 1997.

7. Fredell, R., Vlot A., Verbruggen, M. Fatigue and Residual Strength Characteristics of Fiber Metal Laminates Subjected to Incidental Damage. Report LR-708, TU Delft, Dec.1992.

8. Roebroeks G.H.J.J., and Vogelesang L.B. Fatigue of Fiber- Metal Laminates. 9th Int. Spring Meeting Fatigue of MMC and Multimaterials , Paris, 1990.

9. Alderliesten R.C., Hagenbeek M., Homan J.J., Hooijmeijer P.A., de Vries T.J., Vermeeren C.A.J.R. Fatigue and Damage Tolerance of Glare. Applied Composite Material, Vol. 10: pp. 223-242, 2003.

10. Vogelesang L.B., Vlot A. Development of fibre metal laminates for advanced aerospace structures. Journal of Materials Processing Technology, Vol. 103: pp. 1-5, 2003.

11. Vogelesang, L.B., Gunnink, J.W., Roebroeks, G.H.J.J., Müller, R.P.G. Toward the Supportable and Durable Aircraft Fuselage Structure. Proceedings of the 18th Symposium of the International Committee on Aeronautical Fatigue, Melbourne, Australia, pp. 257-272, 1995

12. Vogelesang, L.B., Schijve, J. Fiber Metal laminates: Damage Tolerant Aerospace Materials. In Case Studies in Manufacturing with Advanced Materials 2, Elsevier, pp.259-260, 1995.

13. Dornheim M.A. Low-Fatigue materials save weigth on A380. Aviation Week \& Space Technology, 18 June 2001.

14. Ohrloff N. and Horst P. Feasibility Study of the Application of Glare materials in wide Body Aircraft Fuselage. 13th European Chapter Conference of SAMPE, Hamburg, pp. 131-142, 1992.

15. de Vries T.J., Vlot A., Hashagen F. Delamination behavior of spliced Fiber Metal Laminates. Part 1. Experimental results. Composite Structures, Vol. 46: pp. 131145, 1999.

16. Hashagen F., de Borst R., de Vries T.J.. Delamination behavior of spliced Fiber Metal Laminates. Part 2. Numerical investigation. Composite Structures, Vol. 46: pp.147-162, 1999.

17. Aliabadi M.H.. The Boundary Element method, Volume 2, Applications in Solids and Structures. John Wiley \& Sons, New York, 2002.

18. Banerjee, P.K., Butterfield, R.. Boundary Element Methods in Engineering Science. McGraw-Hill, Maidenhead, 1981.

19. Davì G., 1989. A general boundary formulation for the numerical solution of bending multilayer sandwich plates. In: Brebbia, C.A. Connor, J.J. (Eds.), Advances in Boundary Elements. Proceedings of the 11th International 
Conference on Boundary Element Methods. Computational Mechanics Publications, Southampton, pp. 25-35.

20. Eshelby, J.D., Read, W.T., Shockley, W. Anisotropic elasticity with applications to dislocation theory. Acta Metallurgica, Vol. 1: pp. 251-259, 1953.

21. Stroh, A.N. Dislocations and cracks in anisotropic elasticity. Philos. Mag., Vol. 8: pp. 625-646, 1958.

22. Ting T.C.T. Anisotropic Elasticity. Theory and Application. Oxford University Press, New York, 1996.

23. Mantic V., Paris F. Integral kernels in the 2-D Somigliana displacement and stress identities for anisotropic materials. Computational Mechanics, Vol.22: pp.77-88, 1998.

24. Denda, M. 2D fundamendal solutions for the general anisotropic solids with computer codes. Electronic Journal with Boundary Elements, Vol. 3: pp. 14-24, 2005.

25. Pan E. A BEM analysis of fracture mechanics in 2D anisotropic piezoelectric solids. Engineering Analysis with Boundary Elements, Vol. 23: pp. 67-76, 1999.

26. Denda M., Mansukh M. Upper and lower bounds analysis of electric induction intensity factors for multiple piezoelectric cracks by the BEM. Engineering Analysis with Boundary Elements, Vol. 29: pp. 533-550, 2005.

27. Lekhnitskii, S.G. Theory of elasticity of an Anisotropic Body. Holden-Day, San Francisco, 1963.

28 Davì, G., Milazzo, A., "Integral equation approach to composite laminate analysis”, Invited paper. Journal of the Chinese Institute of Engineers, Vol. 22: pp. 695-708, 1999.

29. Davì, G., Milazzo, A., "Multidomain boundary integral formulation for piezoelectric materials fracture mechanics”, International Journal of Solids and Structures, Vol. 38: pp. 7065-7078, 2001.

30. Blandford, G.E., Ingraffea, A.R., Ligget, J.A. Two-dimensional stress intensity factor cmputations using boundary element method. International Journal for Numerical Methods in Engineering , Vol. 17: pp. 387-404, 1981.

31. Tan, C.L., Gao, Y.L., Afagh, F.F. Boundary element analysis of interface cracks between dissimilar anisotropic materials. International Journal of Solids and Structures, Vol. 29: pp. 3201-3220, 1992.

32 Aliabadi, M. H.. Boundary element formulations in fracture mechanics. Applied Mechanics Review, Vol. 50 : pp. 83-96, 1997.

33. Thomsen O.T., Rits W, Eaton D.C.G., Brown S. Ply Drop-Off effects in CFRP/Honeycomb sandwich Panels-Theory. Composite Science and Technology, Vol. 56: pp. 407-422, 1996. 